\title{
Damping behavior of bent fiber NSOM probes in water
}

\author{
Rod S. Taylor, a) Dusan Vobornik, Zhengfang Lu, Roderick A. Chisholm, and \\ Linda J. Johnston \\ Steacie Institute for Molecular Sciences, National Research Council of Canada, Ottawa, Ontario K1A OR6, \\ Canada
}

(Received 13 November 2009; accepted 7 January 2010; published online 25 February 2010)

\begin{abstract}
The damping behavior of bent fiber near-field scanning optical microscopy (NSOM) probes operating in tapping mode oscillation is investigated in air and water. We show that the significant drop in probe quality factor $\mathrm{Q}$, which occurs at the air-water interface, is due to meniscus damping. As the probe is immersed in water viscous damping adds to the meniscus damping. Damping effects which lead to a progressive drop in the peak tapping mode resonance frequency are accounted for by additional torsional modes of probe vibration. Understanding the damping processes should lead to the design of high sensitivity NSOM probes for scanning soft biological samples under liquid. (C) 2010 American Institute of Physics. [doi:10.1063/1.3309392]
\end{abstract}

\section{INTRODUCTION}

Near-field scanning optical microscopy (NSOM) is a hybrid scanning probe-optical technique used for overcoming the diffraction limit of light for optical imaging, spectroscopy, and high resolution marking of materials. ${ }^{1-3}$ NSOM is most commonly implemented using optical fiber probes with highly tapered tips. ${ }^{4-6}$ One version of these probes is the bent fiber probe designed to operate as a cantilever in standard atomic force microscopes (AFMs) which use laser deflection distance regulation to control the probe tip on a surface. ${ }^{7,8}$ Over the years these probes have been successfully used in photonic $^{9}$ as well as biological ${ }^{1}$ applications, primarily for dry samples. However, most biological applications require imaging in an aqueous environment. The bent fiber design described in Refs. 7 and 8 seems to offer an advantage over other cantilevered NSOM probes for imaging in fluids since the distance between the NSOM tip and the bend can be made long enough to keep the horizontal portion of the cantilever out of the liquid, thereby avoiding serious damping problems. Cantilevered bent fiber probes can also be operated in the tapping mode (intermittent contact) to minimize shear damage to soft biological samples such as supported membranes and live cells. ${ }^{10}$ We have demonstrated that cantilevered probes can be used to image lipid membranes in an aqueous environment and fixed cells in air, achieving optical resolutions down to $50 \mathrm{~nm} .{ }^{11-13}$ The same probe design has been used to achieve an order of magnitude reduction in the excitation area for fluorescence correlation spectroscopy (FCS) measurements on two-dimensional membrane samples. ${ }^{14}$ Nevertheless, the high spring constant of these probes presents some limitations for routine and rapid scanning of a wide range of biological samples in an aqueous environment without damage. These limitations can be addressed by implementation of a low stiffness probe design such as described in Ref. 15. In this paper we provide a detailed characterization of the damping behavior in water of high and low spring constant bent fiber probes.

\footnotetext{
${ }^{a)}$ Electronic mail: rod.taylor@nrc-cnrc.gc.ca.
}

The presence of a liquid around the probe results in damped tapping mode operation making the probes less sensitive down on a surface. Furthermore, damping can also cause a decrease in the cantilever resonance frequency compared to that in air. ${ }^{16,17}$ Although some aspects of bent fiber NSOM probe damping have been described previously, ${ }^{15,18-20}$ there is a clear need for a thorough study of the damping behavior. The experimental results provided here show the dramatic damping of bent fiber probes as they enter an air-water interface. Although the nature of this damping mechanism has not been investigated for bent fiber probes, one recent paper described the damping behavior of constant diameter nanoneedle probes (diameters $<400 \mathrm{~nm}$ ) at an airliquid interface. ${ }^{21}$ It was suggested that the damping at the interface was due to a surface drag or "squeeze damping" force of the meniscus on the side walls of the probe caused by the large shear rates across the thin meniscus channel. The authors constructed a model for meniscus damping based on capillary and wetting phenomena described in Ref. 22. In this paper we use their model to successfully describe the damping properties of our much larger $(40-70 \mu \mathrm{m})$ tapping mode oscillation bent fiber NSOM probes at the air-liquid interface.

As the probes are further immersed into the water some added viscous damping occurs together with a progressive decrease in the probe's peak resonance frequency. In the past, the decrease in resonance frequency has been attributed to the vibrating structure acting as though it had an extra drag mass produced by the pushing of the liquid. ${ }^{23}$ The decrease in resonance frequency as the probe is lowered into the liquid is measured and compared to the hydrodynamic model of Kirstein et al. ${ }^{17}$ From the comparison we conclude that these bent fiber probes must undergo complicated wobble or torsional modes of vibration perpendicular to the axis of the vertical portion of the probe to account for the observed frequency shift. The detrimental influence of these new vibration modes on NSOM probe resolution will be described. 


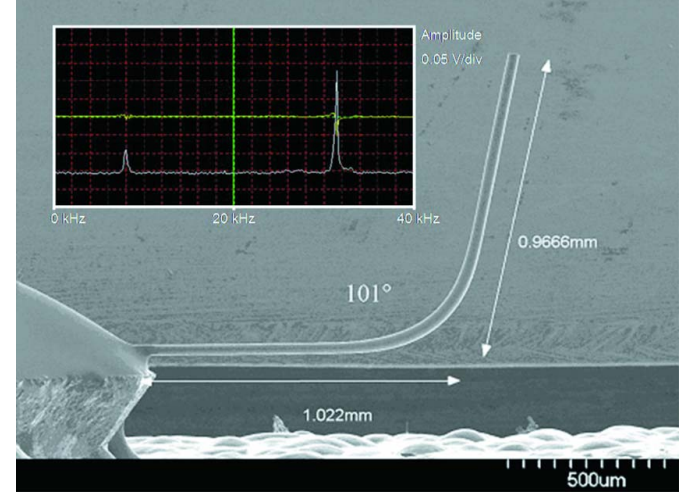

FIG. 1. (Color online) SEM sideview image of a bent, upside down, low-k NSOM probe glue mounted onto an AFM stub. The bend angle is $180^{\circ}$ $-101^{\circ}=79^{\circ}$. The stub is mounted in the AFM at $11^{\circ}$ making the probe tip normal to the sample surface. Inset shows a typical cantilever tuning curve in air indicating the fundamental $\left(\nu_{0}=8 \mathrm{kHz}\right)$ and first higher $\left(\nu_{1}\right.$ $=31 \mathrm{kHz}$ ) resonances.

\section{EXPERIMENTS}

\section{A. Fabrication and characterization of bent fiber NSOM probes}

The probes described in this paper have been used to perform NSOM experiments on a combined AFM/NSOM microscope based on a Veeco Instruments Inc. Bioscope mounted on an inverted fluorescence microscope (Zeiss Axiovert 100) as described previously. ${ }^{24}$ The probes were bent into the shape of a cantilever to accommodate the standard laser deflection distance regulation of the AFM; this allows the probes to be vibrated in the vertical direction in a tapping or intermittent contact mode of AFM operation. A two step chemical etching process was used to reduce the diameter of the fiber as well as to produce a sharp conical tip. The probes were evaporation coated with approximately $400 \mathrm{~nm}$ of aluminum and then focused ion-beam (FIB) milling was used to produce a flat-topped tip with a circular subwavelength optical aperture.

Figure 1 shows a scanning electron microscope (SEM) image of a side view of a mounted low spring constant $(\mathrm{k})$ bent NSOM fiber probe. Both low and high spring constant bent fiber probes with physical dimensions indicated in Table I were tested in air as well as with the vertical portion of the probe submerged up to $500 \mu \mathrm{m}$ in water. The values of $\mathrm{k}$ shown in Table I were calculated from the cantilever dimensions using the formulae provided in the book Scanning Force Microscopy by $\operatorname{Sarid}^{25}$ (Appendix). It is possible to

TABLE I. Cantilever dimensions and fundamental resonance properties in air (calculations made in Appendix).

\begin{tabular}{lccc}
\hline \hline & High-k & High-k (cut) & Low-k \\
\hline Horizontal length & $0.82 \mathrm{~mm}$ & $0.82 \mathrm{~mm}$ & $1.02 \mathrm{~mm}$ \\
Vertical length & $1.35 \mathrm{~mm}$ & $0.675 \mathrm{~mm}$ & $0.97 \mathrm{~mm}$ \\
Probe radius & $35.5 \mu \mathrm{m}$ & $35.5 \mu \mathrm{m}$ & $19 \mu \mathrm{m}$ \\
Calculated k & $494 \mathrm{~N} / \mathrm{m}$ & $494 \mathrm{~N} / \mathrm{m}$ & $22 \mathrm{~N} / \mathrm{m}$ \\
Calculated $\nu_{0}$ & $30.5 \mathrm{kHz}$ & $40.6 \mathrm{kHz}$ & $13.6 \mathrm{kHz}$ \\
Experimental $\nu_{0}$ & $13 \pm 1.3 \mathrm{kHz}$ & $24.7 \pm 1 \mathrm{kHz}$ & $8 \pm 1 \mathrm{kHz}$ \\
\hline \hline
\end{tabular}

decrease the probe spring constant by increasing the horizontal length of the cantilever. However, this length is limited to approximately $1 \mathrm{~mm}$ in order to ensure that the diode laser distance regulation beam, which has very little positioning latitude (i.e., restricted to target $\approx 400 \pm 200 \mu \mathrm{m}$ from the beginning of the cantilever), is as close to the bend as possible to achieve the maximum tip deflection as measured by a root mean square $\left\langle\mathrm{A}^{2}\right\rangle^{1 / 2}$ (rms amplitude) voltage on the Veeco Instruments Inc. Bioscope. Due to this length restriction we chose to reduce the radii $(\mathrm{R})$ of the fibers to lower the spring constant since as indicated in Appendix $\mathrm{k} \propto \mathrm{R}^{4}$. Based on the probe geometries outlined in Table I there is a predicted ratio of $\times 23$ in spring constants between the high and low-k probes.

The cantilever parameters of both the high and low- $\mathrm{k}$ probes deviate significantly from that of an ideal cantilever, i.e., a horizontal cylinder fixed at one end with a small concentrated mass at the other end (see Appendix). The considerable extended mass past the bend results in a wide discrepancy between the calculated (ideal case) and experimental fundamental resonance frequencies as shown in Table I. The calculated values for $\nu_{0}$ become closer to the experimental values when the length of the vertical mass is reduced by a factor of two [i.e., high-k (cut probe)]. Unfortunately it is not straightforward to reduce the length of the vertical portion of the probe for use in our NSOM fluid cell since the length is chosen so that the horizontal portion of the cantilever clears the cell holding the immersed sample. Even if the cell design was modified to have a lower profile we would still need a length of 500-600 $\mu \mathrm{m}$ to accommodate the radiused bend (Fig. 1), which permits high light transmission through to the conical tip. $^{24}$

\section{B. Damping and meniscus measurements}

In the damping experiments we used the AFM to monitor the peak tapping mode resonance frequency together with the full width at half maximum (FWHM) of the resonance distribution. The AFM's stepper motor adjusted the fiber height above a water covered glass surface. During water immersion the drive amplitude of the tapping mode piezoelectric crystal was increased to keep the rms voltage approximately constant (at approximately $0.3 \mathrm{~V}$ ) and at a level which gave good signal to noise in the distance regulation feedback loop. The drive amplitudes were then used to normalize the rms voltages to generate plots of rms voltage versus probe submersion height.

All NSOM damping experiments were conducted in a home-made wet-cell designed to maintain a constant depth of water over long periods of time (i.e., several hours or longer if needed). The wet cell consists of two parts: the bottom part is the sample glass substrate and the top part is a commercially available bio-well cover (HydriWell, HBW20 Grace Bio-laboratories) that provides a several hundred micrometers thick space between the sample and the top cover. Two small holes ( $2 \mathrm{~mm}$ diameter) were punched in the top cover. One hole was used to allow the NSOM probe access to the sample and the other as a supply hole to add water. An excess amount of water $(\sim 150 \mu \mathrm{l})$ forming a large droplet 
was always placed on the supply hole and in this way any evaporation of water during the experiment was compensated by the water in the droplet. We ensured that the water level was maintained over several hours by adding every hour or so (depending on the humidity level in the room) $\sim 50 \mu \mathrm{l}$ of water to the supply droplet. The height of the water above the glass surface was very reproducible over time $(500 \pm 25 \mu \mathrm{m})$ and with different wet cells.

The experimental set-up for independent meniscus measurements of the metalized NSOM probes is as follows. A water cell similar to the NSOM sample cell is used for the measurement. It has a $2 \mathrm{~mm}$ hole as a liquid source for the meniscus measurement and another $1.5 \mathrm{~mm}$ hole for the water supply. With a single drop of water added on the supply hole, the level of the liquid source was kept unchanged over the duration of the experiment. A ninety times stereo microscope with installed micrometer scales and a $360^{\circ}$ protractor was used to observe and measure the height (h) and the contact angle $(\theta)$ of the meniscus, respectively. The contact angle is defined as the angle at the contact line between the air-liquid interface and vertical. Meniscus height is defined as the equilibrium height of the meniscus relative to the planar surface of the liquid. A three-dimensional stage held the bent fiber NSOM probe and moved the probe to the center position of the liquid source as checked by two perpendicularly positioned microscopes. The microscope micrometer scales were calibrated by measuring the thickness of an optical fiber with known diameter $(125 \mu \mathrm{m})$. Millipore filtrated water was used through all the measurements to ensure the same experimental conditions to the damping experiments.

\section{RESULTS AND DISCUSSION}

\section{A. Damping behavior of bent fiber tapping mode oscillation in water \\ 1. Probe quality factor $Q$}

The damping of cantilever oscillation in ideal incompressible fluids is generally characterized by measuring the probe quality factor $(\mathrm{Q})$ defined as the quotient of the peak resonance frequency and the FWHM of the resonance curve (i.e., $\mathrm{Q}=\nu / \Delta \nu_{\mathrm{FWHM}}$ ). The value of $\mathrm{Q}$ changes dramatically depending on the medium in which the cantilever or part of the cantilever is immersed, e.g., Q decreases as damping increases going from vacuum to air to liquid. ${ }^{26}$ It is also determined by the way the fixed end of the cantilever is connected to an AFM stub. Since this is usually accomplished using a glue the damping properties of the glue (stiffness) are very important. We have found that good values of Q [approximately 300 in air $\left(\nu_{1}\right.$ mode) $)$ can be obtained using Loctite E-30CL epoxy. We have also observed that higher Q's $(\times 2)$ can be obtained by operating at the resonance frequency corresponding to the second normal mode $\left(\nu_{1}\right)$ of vibration rather than at the fundamental mode $\left(\nu_{0}\right)$ (Appendix). We hypothesize that this is related to the significant deviation in the bent probe from an ideal cantilever which may favor more efficient oscillation at $\nu_{1}$. Observation of higher Q's at higher eigenfrequencies has also been made in Refs. 27 and 28.
When the last $500 \mu \mathrm{m}$ of the vertical component of the probes are immersed in water the Q-values drop significantly to approximately 35 for the high-k probes and to approximately 13 (2.7 times lower) for the low-k probes. The interaction force $\left(\mathrm{F}_{\text {inter }}\right)$ that a probe tip exerts on a sample surface for a given tip-sample interaction amplitude $\Delta \mathrm{x}$ (based upon cantilever AFM force measurements $\Delta \mathrm{x}$ is taken as approximately $10 \mathrm{~nm}$ ) depends on the probe Q through ${ }^{29}$

$$
\mathrm{F}_{\text {inter }}=\mathrm{k} \Delta \mathrm{x} / \mathrm{Q} \text {. }
$$

According to this formula the lower value of $\mathrm{Q}$ for the low- $\mathrm{k}$ probe unfortunately tends to undermine achieving a much lower interaction force provided by the reduction in spring constants, i.e., the $\times 23$ drop in $\mathrm{k}$ is offset by the $\times 2.7$ decrease in $\mathrm{Q}$ resulting in an overall $\times 8.3$ reduction in the predicted interaction force. The magnitude of the probesurface interaction force is estimated from Eq. (1) to be 17 $\mathrm{nN}$ for the low-k probe.

\section{Probe damping in water}

As the metalized probe tip first makes contact with the water a meniscus rises up from the surface to attach itself to the end of the fiber. Both the Q and the tapping mode rms voltage drop suddenly as shown in Fig. 2(a) for the fundamental mode and Fig. 2(b) for the first higher vibrational mode of a low-k probe. Most of the change $(\times 5)$ in $\mathrm{Q}$ occurs over the first $25 \mu \mathrm{m}$ of submersion. As the probe is further immersed into the water the height of this meniscus decreases to a smaller value which is independent of the probe depth. The Q and rms voltage continue to slowly decrease towards a steady-state value obtained at depths greater than $200 \mu \mathrm{m}$.

The rms voltage is a direct measure of the cantilever amplitude at the point where the diode distance regulation laser beam hits the top metalized surface while the Q measures the effect of damping on increasing the width of the cantilever's resonance curve. Both measurements reveal the dramatic increase in damping followed by a progression to a steady-state value which is determined by the viscous damping of the probe oscillation. Similar overlap of the $\mathrm{Q}$ and rms curves is obtained when one raises the probe from the surface to break through the water-air interface at $\mathrm{H} \sim 500 \mu \mathrm{m}$. However, in this case as more of the vertical part of the probe emerges from the water it effectively drags a progressively larger column of water which adheres to the flat end portion of the probe ${ }^{21}$ (i.e., $\mathrm{h}_{\max } \sim 2$ times fiber diameter) creating the hysteresis effect shown in Fig. 3 for the high-k probe. Figure 3 also shows that the high-k probe exhibits a similar rapid fall-off in $\mathrm{Q}$ as the probe is dropped through the air-water interface although as mentioned above the steadystate value for the $\mathrm{Q}$ is higher than for the low-k probe.

A similar sudden drop in cantilever $\mathrm{Q}$ at an air-liquid interface has been observed using a bent NSOM fiber probe in water ${ }^{15}$ and a bent tungsten AFM probe immersed into liquid paraffin. ${ }^{20}$ In both these experiments the probe oscillation is parallel to the vertical portion of the probe (i.e., tapping or intermittent contact mode) and laser deflection distance regulation occurs from the top surface of the horizontal part of the cantilever. A sudden drop in $\mathrm{Q}$ has also 
(a)

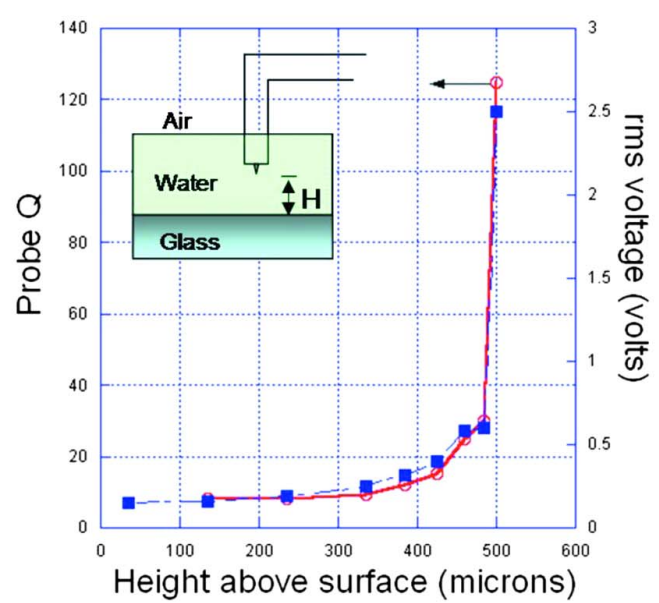

(b)

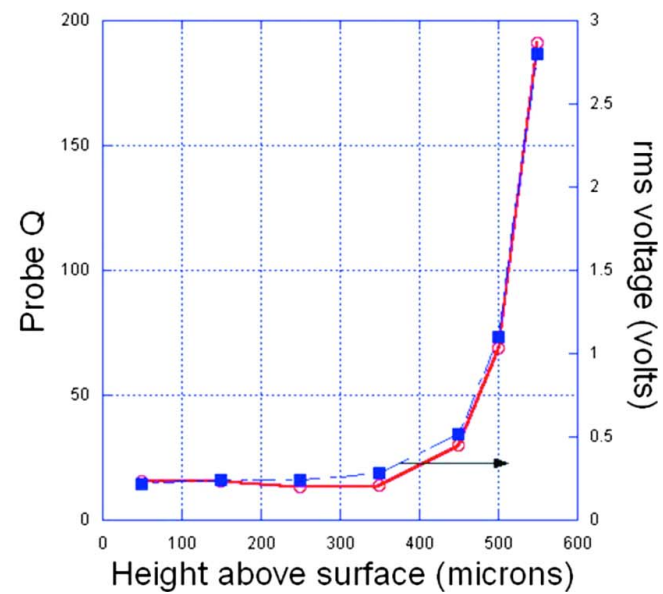

FIG. 2. (Color online) (a) Low-k probe $\mathrm{Q}(\bigcirc)$ and tapping mode $\left(\nu_{0}\right.$ $=8 \mathrm{kHz})$ normalized rms voltage $(\boldsymbol{\square})$ as a function of the probe height $(\mathrm{H})$ above a glass surface. (b) Low-k probe Q $(\bigcirc)$ and tapping mode $\left(\nu_{1}\right.$ $=38 \mathrm{kHz}$ ) normalized rms voltage $(\mathbf{\square})$ as a function of the probe height above the glass surface. The height of the water was $500 \mu \mathrm{m}$.

been observed using a vertically mounted fiber which oscillates perpendicular to the fiber axis. In this case distance regulation is by means of shear force detection. ${ }^{19}$ In Ref. 20 the authors found that the magnitude of the drop in the vibrational amplitude crossing the air-liquid boundary was qualitatively inversely proportional to the sharpness of the tip. In our case we have a sharp conical tip with a height of only $4 \mu \mathrm{m} .^{24}$ This tip sits on a much larger flat-topped cladding region (shown in Fig. 2) of the fiber with a diameter equal to approximately 38 and $71 \mu \mathrm{m}$ for the low and high-k probes, respectively. This diameter is constant over the entire water submersion depth. This essentially flattopped probe endface results in a very sharp transition [estimated from Fig. 2(a) to be $\leq 25 \mu \mathrm{m}$ ] for the drop in Q. The rapid decrease in $\mathrm{Q}$ with submersion depth (D) means that even a small height of water will cause significant deterioration in probe Q. This has implications to liquid cell ${ }^{19}$ and

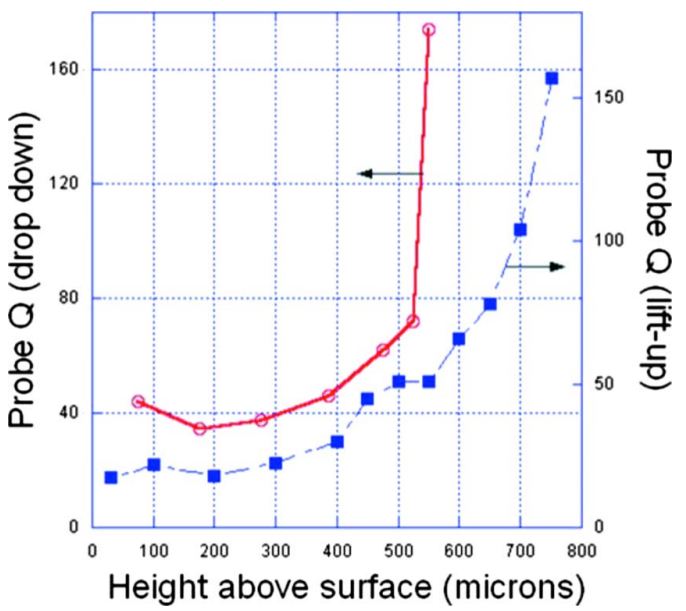

FIG. 3. (Color online) Hysteresis effect (high-k probe) in Q resulting from the difference in $\mathrm{Q}$ obtained when dropping the probe through the air-water interface to a glass surface $(\bigcirc)$ compared to raising it $(\mathbf{\square})$ through the water to the water-air interface (at $\mathrm{H}=500 \mu \mathrm{m}$ ) dragging a approximately $200 \mu \mathrm{m}$ high column of water attached to the probe endface.

probe designs aimed at minimizing the immersion depth to $\leq 100 \mu \mathrm{m}$ or attempting to eliminate the damping effects by creating a diving bell ${ }^{19,30}$ to surround the probe except over the last tens of microns.

\section{B. Meniscus model for probe damping at the air- water interface}

Since the sharp transition in damping occurs very close to the air-water interface it seems reasonable that it is related to the meniscus that forms once probe contact is made with the surface. Ref. 21 has suggested that upon initial contact of their nanoneedle probe tip there appears a surface drag or squeeze damping force of the meniscus on the side walls of the probe caused by the large shear rates across the thin meniscus channel. In a separate experiment described in Sec. II B we measured the meniscus height (h) and contact angle $(\theta)$ for both low-k and high-k metalized probes slowly immersed into water. The results are shown in Table II for the case when the probe first makes contact with the water; when it is partially submerged $(D \geq h)$ and when it has been pulled out of the water to produce a column of water attached to the

TABLE II. Meniscus height and contact angle in water for a metalized NSOM probe.

\begin{tabular}{|c|c|c|}
\hline & $\mathrm{h}(\mu \mathrm{m})$ & $\theta^{\circ}$ \\
\hline \multicolumn{3}{|c|}{ Case A: first contact with water surface } \\
\hline low-k probe & $12 \pm 2$ & $72 \pm 2.5$ \\
\hline high-k probe & $34 \pm 4$ & $72 \pm 2.5$ \\
\hline \multicolumn{3}{|c|}{ Case B: probe partially submerged $(D \geq h)$} \\
\hline low-k probe & $7.5 \pm 2$ & $80 \pm 2.5$ \\
\hline high-k probe & $22 \pm 2.5$ & $82 \pm 2.5$ \\
\hline \multicolumn{3}{|c|}{ Case C: probe just exiting the water } \\
\hline low-k probe & $67 \pm 7$ & $45 \pm 3$ \\
\hline high-k probe & $140 \pm 14$ & $40 \pm 4$ \\
\hline
\end{tabular}


base of the probe. In the latter case the value of (h), which is approximately $140 \mu \mathrm{m}$ for the high-k probe, compares to the value of $200 \mu \mathrm{m}$ obtained from the hysteresis data shown in Fig. 3.

The height $h(\theta)$ of the meniscus surrounding a cylinder is given $b^{21}$

$$
\mathrm{h}(\theta)=\mathrm{R} \cos \theta \ln [2.25 \kappa / \mathrm{R}(1+\sin \theta)],
$$

where $\theta$ is defined as the angle at the contact line between the air-liquid interface and vertical, $\mathrm{R}$ is the fiber radius, and $\kappa$ is the capillary length defined as

$$
\kappa=(\gamma / \rho \mathrm{g})^{1 / 2} .
$$

In Eq. (3) $\gamma$ is the liquid surface tension and $\rho$ the density of the liquid and $\mathrm{g}$ is the acceleration due to gravity. For water at room temperature $\gamma=72 \times 10^{-3} \mathrm{~N} / \mathrm{m}, \rho=1 \times 10^{3} \mathrm{Kg} / \mathrm{m}^{3}$, and $\mathrm{g}=9.8 \mathrm{~m} / \mathrm{s}^{2}$ resulting in $\kappa=2.7 \mathrm{~mm}$, a length which is long compared to the fiber diameter and meniscus height. Using our measured value for $\theta=80^{\circ}$ (see Table II) Eq. (2) predicts $\mathrm{h}=16.4 \mu \mathrm{m}$ for the low-k probe with case B immersion (Table II). For the high-k probe $\theta=82^{\circ}$ and Eq. (2) predicts $\mathrm{h}=22.1 \mu \mathrm{m}$. Experimentally (Table II) we observe $\mathrm{h}=7.5$ and $22 \mu \mathrm{m}$, respectively.

When the fiber is submerged a depth $\mathrm{D}$ in the liquid, viscous drag forces $F_{d}$ act on the vibrating fiber. The probe velocity (v) in the vertical vibration direction is equal to the quotient of the peak to peak amplitude (2A) (obtained from AFM force curve measurements: for the low-k probe $2 \mathrm{~A}$ $=148 \mathrm{~nm}$; for the high $-\mathrm{k}$ probe $2 \mathrm{~A}=320 \mathrm{~nm}$ ) and the resonance period $\left(1 / \nu_{0}\right)$. The value for $\mathrm{v}$ is small (i.e., low- $\mathrm{k}$ probe $\mathrm{v} \approx 1.2 \times 10^{-3} \mathrm{~m} / \mathrm{s}$; for the high-k probe $\mathrm{v} \approx 4.2$ $\times 10^{-3} \mathrm{~m} / \mathrm{s}$ ) guaranteeing a low Reynolds number (Re) and laminar flow since for water $\operatorname{Re} \approx \rho \mathrm{vL} / \eta=2$ (high-k case), where $\mathrm{L}$ is the flow interaction length $(500 \mu \mathrm{m})$ and $\eta$ is the dynamic viscosity $\left(1 \mathrm{cP}\right.$ or $\left.1 \times 10^{-3} \mathrm{~Pa} \mathrm{~s}\right)$. The normalized viscous drag force is given in Ref. 21 as

$$
\begin{aligned}
\mathrm{F}_{\mathrm{d}} / \mathrm{v}= & 2 \pi \eta \mathrm{D} /[\ln (\mathrm{D} / 2 \mathrm{R})-0.2+1.8 \mathrm{R} / \mathrm{D}], \\
& \text { valid for } \mathrm{D} \geq 4 \mathrm{R} .
\end{aligned}
$$

For the low-k probe immersed $\mathrm{D}=500 \mu \mathrm{m}, \mathrm{F}_{\mathrm{d}} / \mathrm{v}=1.3$ $\times 10^{-6} \mathrm{~N} \mathrm{~s} / \mathrm{m}$ corresponding to a drag force of $1.5 \mathrm{nN}$, while for the high-k probe $\mathrm{F}_{\mathrm{d}}=6.9 \mathrm{nN}$.

The authors in Ref. 21 also define a novel meniscus damping term analogous to the viscous term

$$
\mathrm{F}_{\mathrm{m}} / \mathrm{v}=2 \pi \mathrm{R} \times 3 \eta \ln (\mathrm{h} / \mathrm{a}) / \theta,
$$

where "a" is a molecular cut-off length (chosen as $1 \mathrm{~nm}$ ), which is used to avoid a singularity in the solution method. ${ }^{21}$ The meniscus damping force is directly proportional to the product of the fiber radius and the viscosity and inversely proportional to the meniscus contact angle. It is only weakly dependent on the meniscus height through $\ln (\mathrm{h} / \mathrm{a})$. For the low-k probe using experimental values for $\mathrm{h}=7.5 \mu \mathrm{m}$ and $\theta=1.39 \mathrm{rad}$. Equation (5) predicts $\mathrm{F}_{\mathrm{m}}=2.7 \mathrm{nN}$ nearly two times larger than the viscous damping force experienced by the probe at a depth of $\mathrm{D}=500 \mu \mathrm{m}$. For the high-k probe $\mathrm{F}_{\mathrm{m}}=19 \mathrm{nN}$ some three times larger than the viscous damping force (at $\mathrm{D}=500 \mu \mathrm{m})$. The increased dominance of me- niscus damping over viscous damping with the high-k probe is consistent with the relatively small drop in $\mathrm{Q}$ with submersion (Fig. 3) compared to the low-k probe (Fig. 2). These calculations indicate that even though the meniscus height is considerably smaller than most of the probe immersion depths the meniscus damping effect is substantial since it acts through the shear rate term " $\theta$ " in Eq. (5) over millimeter distances defined by the capillary length $(\kappa)$.

The viscous damping factor is defined as $1 / \mathrm{Q}_{\mathrm{v}}$ $=\left(F_{d} / v\right) /(m k)^{1 / 2}$, where $m$ is the effective mass of the cantilever and $\mathrm{k}$ is the spring constant. One can use Eq. (4) with $\eta=1.8 \times 10^{-2} \mathrm{cP}$ for the case of probe immersion in air and with a total probe flow interaction length of $2 \mathrm{~mm}$ to estimate the viscous damping effect of air on probe oscillation before probe immersion. Using Eq. (5) we then calculate the ratio of the meniscus damping at the water/air interface $\left(1 / \mathrm{Q}_{\mathrm{m}}\right)$ to the viscous damping factor in air $\left(1 / Q_{v}^{\text {air }}\right)$ to be $Q_{v}^{\text {air }} / Q_{m}$ $=39$. Experimentally we typically observe a $\mathrm{Q}$ ratio equal to 5 . This discrepancy might be explained by the fact that there are other damping effects for probe oscillation in air such as damping of the cantilever at the glue joint as mentioned in Sec. III A 1. It is also possible that the meniscus damping model overestimates probe damping at the water/air interface. This will be discussed later.

The combined damping effect in water is given by the sum of the meniscus damping plus the viscous damping and can be approximated by

$$
\begin{aligned}
1 / \mathrm{Q}_{\mathrm{m}}+1 / \mathrm{Q}_{\mathrm{v}} \approx & (6 \pi \eta \mathrm{R}) \mathrm{x} \ln (\mathrm{h} / \mathrm{a}) / \theta(\mathrm{mk})^{1 / 2} \\
& +2 \pi \eta \mathrm{D} /\left[(\mathrm{mk})^{1 / 2} \ln (\mathrm{D} / 2 \mathrm{R})\right] .
\end{aligned}
$$

For the low- and high-k probe cases the sums are

$$
\begin{aligned}
1 / \mathrm{Q}_{\mathrm{m}}+1 / \mathrm{Q}_{\mathrm{v}} \approx & 9 \times 10^{-3}+2.45 \times 10^{1} \mathrm{xD} / \ln (2.63 \\
& \left.\times 10^{4} \mathrm{xD}\right) \quad \text { low-k, } \\
1 / \mathrm{Q}_{\mathrm{m}}+1 / \mathrm{Q}_{\mathrm{v}} \approx & 1.8 \times 10^{-3}+2.45 \mathrm{D} / \ln (1.4 \\
& \left.\times 10^{4} \mathrm{xD}\right) \quad \text { high-k, }
\end{aligned}
$$

with the submersion depth $\mathrm{D}$ in $(\mathrm{m})$.

In summary the damping model predicts a large drop in Q similar to that observed at the air-water interface shown in Figs. 2 and 3. The model also predicts that the magnitude of both the meniscus and viscous damping (at a given D) is approximately five times larger for the low-k probes than the high-k probes largely due to the $(\mathrm{mk})^{1 / 2}$ term in $1 / \mathrm{Q}$. Consistent with these predictions we observe that the high- $\mathrm{k}$ probe undergoes less meniscus damping compared to the low-k probe resulting in higher $\mathrm{Q}$ when just submerged as well as less viscous damping giving a higher $\mathrm{Q}$ at a depth $\mathrm{D}=500 \mu \mathrm{m}$. For low-k probes Eq. (7) predicts that the magnitude of viscous damping should equal that of the meniscus damping at a submersion depth of $\mathrm{D}=1.2 \mathrm{~mm}$. For the high-k case Eq. (8) predicts that this should occur at a depth $\mathrm{D}=2.7 \mathrm{~mm}$ equal to the capillary length calculated from Eq. (3). Experimentally (Figs. 2 and 3) we observe a faster falloff in Q at depths of only a few hundreds of microns. The model's underestimation for the drop in $\mathrm{Q}$ with $\mathrm{D}$ for both low and high-k probes may be due to an overestimation of 


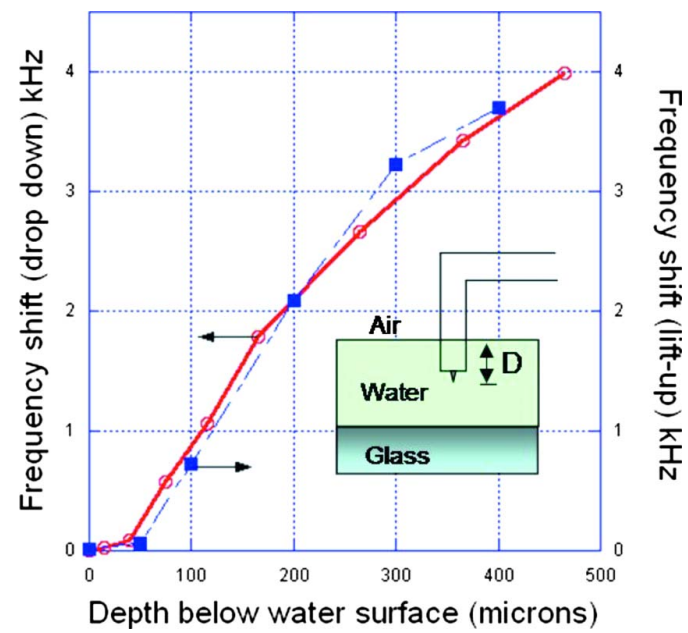

FIG. 4. (Color online) Frequency decrease in the peak resonance frequency ( $38 \mathrm{kHz}$ for the $\nu_{1}$ mode) as a function of depth (D) below the water-air interface for the low-k probe for both drop-down $(\bigcirc)$ and lift-up $(\mathbf{\square})$ modes.

the magnitude of the meniscus damping by a factor of 2 to 3 relative to the viscous damping term. Further work is required to understand the details of meniscus damping. We shall also see in Sec. III C that damping effects may involve more complex oscillations of the probe rather than the simple vertical vibration provided by tapping mode operation.

\section{Decrease in probe resonance frequency with water submersion}

As the probe enters the water to a depth $\geq 25 \mu \mathrm{m}$ we begin to observe a small decrease in the peak cantilever resonance frequency. Figure 4 shows that the frequency shift $\left(\nu_{1}\right.$ mode) relative to that obtained in air is more or less linear (slope $=1 \mathrm{kHz}$ per $100 \mu \mathrm{m}$ of immersion) with the depth of water submersion regardless of whether the probe is dropped to the glass surface (drop-down mode) or raised up (lift-up mode) from the surface to the water-air interface. However, the curves appear to level off at larger depth values. A similar $10 \%$ drop in resonance frequency (not shown) has been observed with the fundamental vibrational mode $\left(\nu_{0}\right)$. The rather strong dependence of frequency shift on probe immersion shown in Fig. 4 demonstrates the importance of maintaining a minimum and constant water level to avoid experiencing drifting in the peak resonance frequency.

Frequency shifts up to $20 \%$ have been reported using both NSOM (Refs. 17 and 18) and AFM (Ref. 17) probes totally immersed in water. Numerous theoretical treatments of forced damped harmonic oscillation of cantilevers vibrating in incompressible liquids with a velocity $\delta u / \delta t$ have been described in the literature. Many of these theories solve the linearized Navier-Stokes equations to account for viscous damping. However none of the various theoretical treatments of probe damping treat our case of partial submersion of the vertical part of a vertically vibrating (tapping mode) cantilever.

In the past a hydrodynamic argument is usually presented to explain the frequency shift with probe immersion. ${ }^{17}$ The argument is generally applied to the case where the vi- bration in the liquid is normal to the long axis of the probe. Then to first order the vibrating structure behaves as though an extra drag mass (also refered to as a virtual or comoving mass $\mathrm{m}_{\mathrm{a}}$ ) of fluid is added to the vibrating structure. ${ }^{23}$ This results in an additional inertial force $\mathrm{F}_{\mathrm{i}}=-\mathrm{m}_{\mathrm{a}} \delta^{2} u / \delta t^{2}$. The added mass is proportional to the displaced or pushed mass of fluid $m_{d}$, which is given as the product of the volume $(\mathrm{V})$ of the immersed body (with density $\rho_{\mathrm{b}}$ ) and the density of the fluid $\rho_{\mathrm{f}}$

$$
\mathrm{m}_{\mathrm{a}}=\mathrm{C}_{\mathrm{m}} \mathrm{m}_{\mathrm{d}}=\mathrm{C}_{\mathrm{m}} \rho_{\mathrm{f}} \mathrm{V},
$$

where $\mathrm{C}_{\mathrm{m}}$ is the added mass coefficient, which for an ideal fluid $\mathrm{C}_{\mathrm{m}} \approx 1 .{ }^{17}$ Therefore the value of this added mass is generally approximated as the mass of the volume of water displaced by the submerged portion of the probe. The decrease in frequency follows from the simple $\omega=\left(\mathrm{k} / \mathrm{m}_{\mathrm{eff}}\right)^{1 / 2}$ relationship, in which the effective mass $\mathrm{m}_{\mathrm{eff}}=\mathrm{m}_{\text {tot }}+\mathrm{m}_{\mathrm{a}}$, where $\mathrm{m}_{\mathrm{tot}}$ is the total mass of the bent fiber probe. For the limiting case of a totally submerged probe the ratio of the frequencies measured in fluid compared to that in air is given by

$$
\begin{aligned}
\nu_{\mathrm{f}} / \nu_{\mathrm{a}} & =\left[\mathrm{m}_{\mathrm{tot}} /\left(\mathrm{m}_{\mathrm{tot}}+\mathrm{m}_{\mathrm{a}}\right)\right]^{1 / 2}=1 /\left(1+\mathrm{C}_{\mathrm{m}} \rho_{\mathrm{f}} / \rho_{\mathrm{b}}\right)^{1 / 2} \\
& \approx 1 /\left(1+\rho_{\mathrm{f}} / \rho_{\mathrm{b}}\right)^{1 / 2} .
\end{aligned}
$$

For a fused silica probe $\left(\rho_{\mathrm{b}}=2.2 \times 10^{3} \mathrm{~kg} / \mathrm{m}^{3}\right)$ immersed in water with $\rho_{\mathrm{w}}=1.0 \times 10^{3} \mathrm{~kg} / \mathrm{m}^{3}$ the predicted frequency ratio is 0.83 corresponding to a maximum decrease in resonance frequency of $17 \%$.

In our case probe vibration is along the axis of the submerged portion of the probe (tapping mode) rather than perpendicular to it. The pushing on the water should be through the bottom of the probe (with radius $\mathrm{R}$ ) and the effective volume of the displaced body of water should be $\approx 2 / 3 \pi R^{3}$ which will be unchanged as the probe is lowered into the water. In other words, damping of this piezo-driven vertical tapping mode vibration cannot account for the experimentally observed progressive frequency shift. It would appear that our probe vibration is not confined to just vertical oscillation and must include some torsional motion, i.e., causing motions perpendicular to the axis of the vertical portion of the probe. Using a similar bent low spring constant fiber probe Muramatsu et al. ${ }^{15}$ observed that dragging the probe tip on a surface in contact mode could also excite such torsional modes of oscillation. One can define a torsional spring constant $\left(\mathrm{k}_{\mathrm{tor}}\right)$ analagous to the formula to calculate the cantilever spring constant [Eq. (2) in Appendix]

$$
\mathrm{k}_{\mathrm{tor}}=\pi \mathrm{GR}^{4} / 2 l_{\text {hor }} \mathrm{x} l_{\mathrm{vert}}^{2},
$$

where $\mathrm{G}$ is the shear modulus of glass $\left(3.12 \times 10^{10} \mathrm{~Pa}\right.$ for silica); $\mathrm{R}$ is the probe radius, $l_{\text {hor }}$ is the horizontal length of the probe and $l_{\text {vert }}$ is the length of the vertical component of the bent probe. For our low-k probe $\mathrm{R}=19 \mu \mathrm{m}, l_{\text {hor }}$ $=1 \mathrm{~mm}$, and $l_{\text {vert }}=0.97 \mathrm{~mm}$. Substitution of these values yields a $\mathrm{k}_{\text {tor }}=7 \mathrm{~N} / \mathrm{m}$ which is three times smaller than the spring constant for vertical oscillation (Table I) supporting the argument that the probe can be excited by the piezoelectric driving element to oscillate perpendicular to its vertical axis and it is the damping of this vibration that causes the 


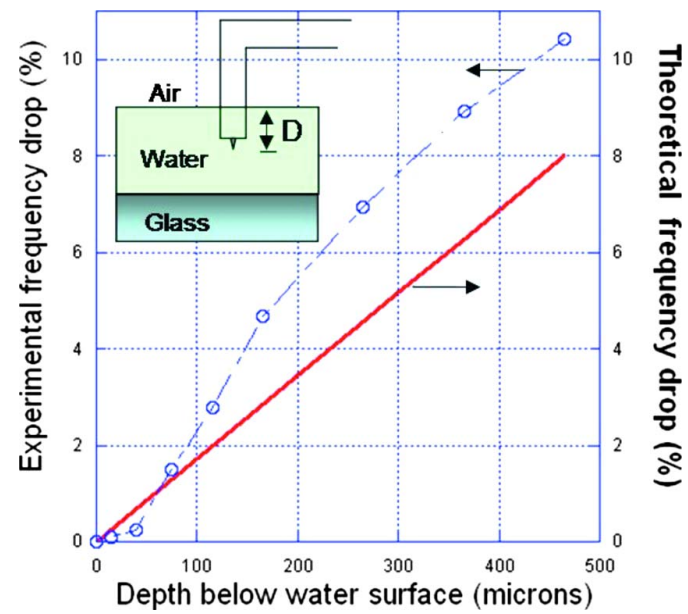

FIG. 5. (Color online) Experimental frequency decrease $(\bigcirc)$ with water immersion as a percentage of the peak resonance frequency for the $\nu_{1}$ mode $\left(\nu_{1}=38 \mathrm{kHz}\right)$ in air (drop down mode) and theoretical prediction (-) using Eq. (13) as a function of probe depth below the water surface for the low-k probe.

frequency shift. Specifically during water immersion damping of the complex set of probe vibrations leads to progressive reshaping of the resonance curves resulting in a decrease in the peak resonance frequency. We therefore use the model of Kirstein et al. ${ }^{17}$ and assume that the added mass $\mathrm{m}_{\mathrm{a}}$ $=\rho_{\mathrm{w}} \mathrm{V}$ (cylinder of radius $\mathrm{R}$ and length $\left.\mathrm{D}\right)=\rho_{\mathrm{w}} \pi \mathrm{R}^{2} \mathrm{D}$. Then it follows that

$$
\begin{aligned}
\omega & =\left(\mathrm{k} / \mathrm{m}_{\text {eff }}\right)^{1 / 2}=\left[\mathrm{k} /\left(\mathrm{m}_{\mathrm{tot}}+\mathrm{m}_{\mathrm{a}}\right)\right]^{1 / 2} \\
& =\left[\mathrm{k} / \mathrm{m}_{\mathrm{tot}} /\left(1+\rho_{\mathrm{w}} \pi \mathrm{R}^{2} \mathrm{D} / \mathrm{m}_{\mathrm{tot}}\right)\right]^{1 / 2} .
\end{aligned}
$$

Since D is generally much less than the total length of the cantilevered probe then $\mathrm{m}_{\mathrm{a}} / \mathrm{m}_{\text {tot }} \ll 1$ and Eq. (12) can be approximated using Macluarin series expansion to give

$$
\omega=\omega_{0}\left(1-\pi \rho_{\mathrm{w}} \mathrm{R}^{2} \mathrm{D} / 2 \mathrm{~m}_{\mathrm{tot}}\right),
$$

i.e., the frequency decrease depends linearly on the depth of probe immersion (D). Substitution for the various parameters in Eq. (13) results in $\omega=\omega_{0}\left(1-10^{-6} \mathrm{D} / 2 \mathrm{~m}_{\text {tot }}\right)$. In Appendix we show that $\mathrm{m}_{\text {tot }}$ for the low-k probe equals $3.0 \times 10^{-9} \mathrm{~kg}$ and therefore $\omega=\omega_{0}\left(1-1.66 \times 10^{-4} \mathrm{D}\right)$, where the depth of probe immersion in the water is in microns. Figure 5 shows a plot of the experimental percentage frequency drop (probe drop down mode) as a function of depth below the water surface as well as the percentage change predicted from Eq. (13). The simple calculation for the frequency decrease predicts the basic linear dependence of frequency shift in immersion depth but underestimates the inertial damping force by roughly $\times \sqrt{2}$ which is equivalent to a $40 \%$ increase in the added mass.

A similar calculation can be made for the cut high-k probe described in Table $\mathrm{I}$ and in Appendix. In this case $\mathrm{m}_{\text {tot }}=0.76 \times 10^{-8} \mathrm{~kg}$ and Eq. (13) becomes

$$
\omega=\omega_{0}\left[1-2.6 \times 10^{-4} \mathrm{D}(\mu \mathrm{m})\right] .
$$

Figure 6 shows that the agreement between experiment and theory [Eq. (14)] is much closer for this probe which should have less torsional oscillation, i.e., due to a smaller vertical

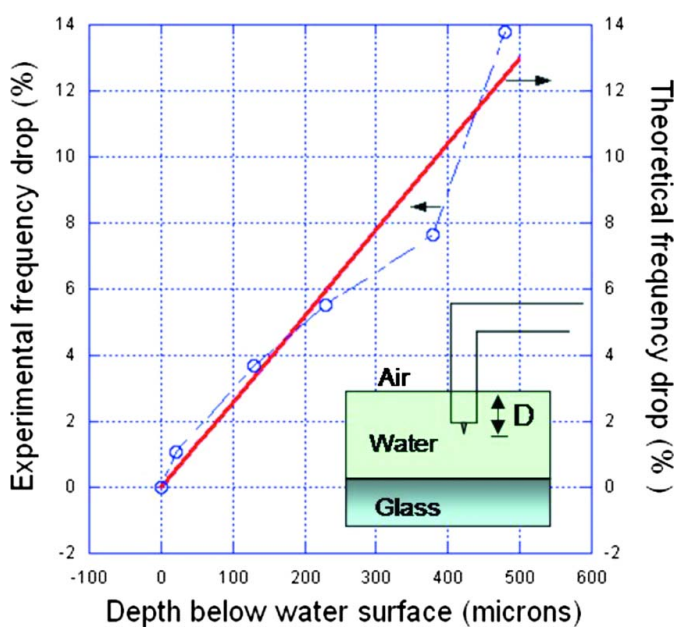

FIG. 6. (Color online) Experimental $(\bigcirc)$ frequency decrease with water immersion as a percentage of the peak resonance frequency of the $\nu_{0}$ mode in air $\left(\nu_{0}=24.7 \mathrm{kHz}\right)$ and theoretical prediction (-) using Eq. (14) as a function of probe depth below the water surface for the $c u t$ high-k probe.

length $\left[l_{\text {vert }}\right.$ in Eq. (11) $]$ compared to the longer low-k probe.

There are two important implications of nonvertical NSOM probe vibration whether it occurs in air or under a liquid. The torsional vibration will produce shear force interaction of the probe tip and the sample which may cause damage and diminish the benefit of tapping mode operation. Second the effective probe aperture may be larger than the actual aperture size by approximately the magnitude of the torsional oscillation. This could be especially important for small sub-100 nm NSOM apertures. We are actively investigating the effect of torsional vibration on probe performance. Figure 7 shows preliminary results on the effect of low-k probe wobble in air at decreasing the AFM resolution on an AFM test grid consisting of $1 \mu \mathrm{m}$ squares. The NSOM probe was identical to those described in this paper but was not focused-ion beam milled and therefore had a sharp tip. Figure 7 provides a comparison of probe AFM resolution

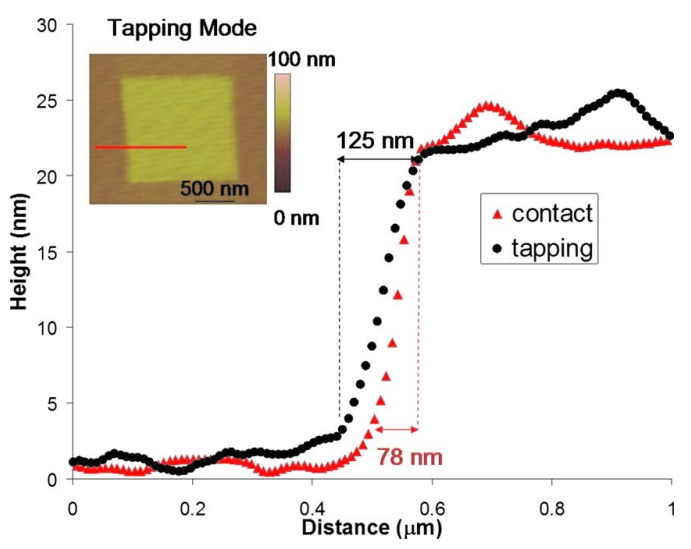

FIG. 7. (Color online) Topography image (inset) of a nominal $20 \mathrm{~nm}$ high, $1 \times 1 \mu \mathrm{m}$ square AFM calibration grating. The image was obtained with a non-FIB machined low-k NSOM probe in tapping mode. The line represents the location where a topographic sectional analysis was performed of the step edge with the NSOM probe operated in tapping (circles) and contact mode (triangles) feedback, demonstrating an increased effective probe aperture (10\%-90\% points) while operating in tapping mode. 
with tapping mode (similar conditions to those used in the damping experiments) and contact mode (no oscillation). In agreement with our damping analysis tapping mode operation does lead to poorer topographical resolution (a change of approximately $47 \mathrm{~nm}$ ) due to an estimated $23 \mathrm{~nm}$ wobble amplitude of the probe aperture. We are currently making a comparison of tapping mode versus contact mode probe resolution in water. However, torsional probe oscillation may be similar in air and water. In water torsional oscillations are highly damped relative to that experienced in air, however, the serious probe damping in water results in the use of higher piezoelectric drive amplitudes (up to $\times 10$ ) to keep the rms voltage high (i.e., to keep a good signal to noise ratio in the laser deflection distance regulation feedback loop). Higher drive amplitudes are expected to more efficiently excite the torsional vibrational modes.

\section{CONCLUSION}

We have performed detailed measurements on the damping behavior of tapping mode, bent fiber NSOM probes at the air-water interface and with the probe immersed up to a depth of $500 \mu \mathrm{m}$. We conclude that the meniscus damping model of Yazdanpanah et al. ${ }^{21}$ predicts the basic damping behavior of these probes at the interface. Attempts to reduce the meniscus damping by reducing the meniscus gradient [i.e., higher $\theta$ in Eq. (5)] could result in higher probe Q's and more sensitive AFM-NSOM operation on soft sample surfaces. This might be accomplished by coating the vertical part of the probe with a nonwetting compound. The progressive drop in the probe's peak resonance frequency as it is submerged in water can be accounted for using the model of Kirstein et al. ${ }^{17}$ with the realization that the bent fiber probe, when piezoelectrically driven vertically in the tapping mode, results in torsional modes of oscillation, the damping of which accounts for the frequency decrease. Further investigation of these torsional modes is required to assess their impact on probe sensitivity and probe resolution. Preliminary experiments show that removing the torsional modes of vibration by operating in the contact mode can lead to higher NSOM probe resolution. Unfortunately scanning soft samples in the contact mode is difficult usually resulting in either sample and/or probe damage. However, FCS-NSOM experiments on phase separated bilayers ${ }^{14}$ can be performed in the contact mode once tapping mode imaging is used to locate the region of interest. We are currently investigating whether improved optical resolution results from contact mode operation in these experiments.

\section{APPENDIX: CALCULATIONS OF PROBE SPRING CONSTANT AND RESONANCE FREQUENCIES IN AIR}

We have used Sarid ${ }^{25}$ to calculate $\mathrm{v}_{0}$ (fundamental) and $\mathrm{v}_{1}$ (first higher mode) for a straight solid cylindrical lever free to oscillate at one end, i.e., equivalent to a low-k fiber probe with effective length $l=1.46 \mathrm{~mm}$ and probe radius $\mathrm{R}$ $=18 \mu \mathrm{m}$ and with no vertical (concentrated mass). In the case of the fundamental resonance frequency $\omega_{0}$ equals

$$
\omega_{0}=\left(\mathrm{k} / 0.24 \mathrm{~m}_{\mathrm{eff}}\right)^{1 / 2}=\left[\left(3 \pi \mathrm{ER}^{4} / 4 l^{3}\right) / 0.24\left(\pi \mathrm{R}^{2} l \rho\right)\right]^{1 / 2} .
$$

In this expression $\mathrm{k}$ is the effective cantilever spring constant given by

$$
\mathrm{k}=3 \pi \mathrm{ER}^{4} / 4 l^{3},
$$

and $\mathrm{m}_{\mathrm{eff}}$ is the effective cantilever mass equal to the product of the cylinder volume $\left(\pi \mathrm{R}^{2} l\right)$ and the cantilever density $(\rho)$. In the above equations $\mathrm{E}$ is Young's modulus for the cantilever. For fused silica $E=7.3 \times 10^{10} \mathrm{~N} / \mathrm{m}^{2}$ and density $\rho$ $=2.2 \times 10^{3} \mathrm{~kg} / \mathrm{m}^{3}$. We calculate $\mathrm{k}=5.8 \mathrm{~N} / \mathrm{m}$ and $\omega_{0}=8.6$ $\times 10^{4} \mathrm{rad} / \mathrm{s}$ corresponding to $\nu_{0}=\omega_{0} / 2 \pi=13.69 \mathrm{kHz}$. Experimentally we observed the resonance frequency to be very close, i.e., $13.88 \mathrm{kHz}$ validating Sarid's formulae for the case of a straight optical fiber glued at one end to an AFM stub. From Sarid $^{25}$ the ratio of normal mode frequencies for a rectangular cantilever is $\left(\mathrm{v}_{1} / \mathrm{v}_{0}\right)=(4.694 / 1.875)^{2}=6.27 \mathrm{im}$ plying that the frequency of the next normal mode $\mathrm{v}_{1}$ $=102.72 \mathrm{kHz}$. Experimentally we observe $81.62 \mathrm{kHz}$ (ratio of 5.9), i.e., approximately $20 \%$ lower than predicted. The measured probe quality factor Q defined as $\nu / \Delta \nu$, where $\Delta \nu$ is the FWHM of the cantilever resonance curve for this near ideal cantilever was 350 .

We have also used Sarid's formulism to calculate $\mathrm{v}_{0}$ and $\mathrm{v}_{1}$ for a bent low-k fiber (cantilever parameters outlined in Table I) with an effective distributed length $l$ of $1.0 \mathrm{~mm}$ and distributed mass of $\mathrm{m}_{\mathrm{d}}=\pi \mathrm{R}^{2} l \rho$ and with a fiber radius (R) of $19 \mu \mathrm{m}$. We assume that the additional mass of the vertical part $\left(l_{\mathrm{ver}}=0.97 \mathrm{~mm}\right)$ of the fiber acted as a concentrated mass $\left(\mathrm{m}_{\mathrm{c}}\right)$ suspended at the open end of the cylinder. In this case:

$$
\begin{aligned}
\omega_{0} & =\left(\mathrm{k} / \mathrm{m}_{\mathrm{tot}}\right)^{1 / 2}=\left[\mathrm{k} /\left(\mathrm{m}_{\mathrm{c}}+0.24 \mathrm{~m}_{\mathrm{d}}\right)\right]^{1 / 2} \\
& =\left[\left(3 \pi \mathrm{ER}^{4} / 4 l^{3}\right) /\left(\mathrm{m}_{\mathrm{c}}+0.24 \mathrm{~m}_{\mathrm{d}}\right)\right]^{1 / 2} .
\end{aligned}
$$

We estimate that $\mathrm{k}=22 \mathrm{~N} / \mathrm{m}$ and $\mathrm{m}_{\mathrm{c}}=\pi \rho \mathrm{R}^{2} \times l_{\text {ver }}=2.42$ $\times 10^{-9} \mathrm{~kg}$ and $\mathrm{m}_{\mathrm{d}}=2.5 \times 10^{-9} \mathrm{~kg}$ (i.e., $\mathrm{m}_{\text {tot }}=\mathrm{m}_{\mathrm{c}}+0.24 \mathrm{~m}_{\mathrm{d}}$ $=3.0 \times 10^{-9} \mathrm{~kg}$ ). Therefore $\omega_{0}=85.6 \mathrm{krad} / \mathrm{s}$ and $\nu_{0}$ $=13.6 \mathrm{kHz}$. Experimentally based upon data from numerous probes $\nu_{0}=8 \pm 1 \mathrm{kHz}$ (Table I).

For the high-k probe with dimensions outlined in Table I we get $\mathrm{m}_{\mathrm{c}}=1.18 \times 10^{-8} \mathrm{~kg}, 0.24 \mathrm{~m}_{\mathrm{d}}=0.17 \times 10^{-8} \mathrm{~kg}$ and $\mathrm{m}_{\mathrm{tot}}=1.35 \times 10^{-8} \mathrm{~kg}$ yielding $\mathrm{k}=494 \mathrm{~N} / \mathrm{m}$ and $\nu_{0}$ $=30.5 \mathrm{kHz}$. Experimentally we obtained $\nu_{0}=13 \mathrm{kHz}$ (Table I). A calculation of $\nu_{0}$ was also made for the high-k probe with the vertical part of the fiber cut in half to a length of $0.675 \mathrm{~mm}$. In this case $\mathrm{m}_{\mathrm{c}}=2.2 \times 10^{3} \times 3.14 \times(3.55$ $\left.\times 10^{-5}\right)^{2} \times 0.67510^{-3}=0.588 \times 10^{-8} \mathrm{~kg}$ and with $0.24 \mathrm{~m}_{\mathrm{d}}$ $=0.17 \times 10^{-8} \mathrm{~kg}$ gives $\mathrm{m}_{\mathrm{tot}}=0.76 \times 10^{-8} \mathrm{~kg}$ and $\nu_{0}$ $=40.6 \mathrm{kHz}$. Experimentally we observed (Table I) $\nu_{0}$ $=24.7 \mathrm{kHz}$. Not surprisedly Sarid's formulae for ideal cantilevers overestimate the experimental bent fiber resonance frequencies by up to a factor of $\times 2$ with the greatest discrepancies corresponding to the largest suspended mass, i.e., highest $\mathrm{m}_{\mathrm{c}} / \mathrm{m}_{\mathrm{d}}$ ratio.

${ }^{1}$ R. C. Dunn, Chem. Rev. (Washington, D.C.) 99, 2891 (1999).

${ }^{2}$ A. Lewis, A. Taha, A. Strinkovski, A. Manevitch, A. Khatchatouriants, R. Dekheter, and E. Amman, Nat. Biotechnol. 21, 1378 (2003). 
${ }^{3}$ B. De Lange, F. Cambi, R. Huijbens, B. de Bakker, W. Rensen, M. GarciaParajo, N. van Hulst, and C. Figdor, J. Cell Sci. 114, 4153 (2001).

${ }^{4}$ E. Betzig, J. K. Trautman, T. D. Harris, J. S. Weiner, and R. L. Kostelak, Science 251, 1468 (1991)

${ }^{5}$ G. A. Valaskovic, M. Holton, and G. H. Morrison, Appl. Opt. 34, 1215 (1995).

${ }^{6}$ D. Courjon, K. Sarayeddine, and M. Spajer, Opt. Commun. 71, 23 (1989).

${ }^{7}$ R. S. Taylor, K. E. Leopold, M. Wendman, G. Gurley, and V. Elings, Rev. Sci. Instrum. 69, 2981 (1998).

${ }^{8}$ C. E. Talley, G. A. Cooksey, and R. C. Dunn, Appl. Phys. Lett. 69, 3809 (1996).

${ }^{9}$ R. S. Taylor and K. E. Leopold, Microscopy and Analysis 5, 15, (1999)

${ }^{10}$ H. Muramatsu, N. Chiba, K. Homma, K. Nakajima, T. Ataka, S. Ohta, A. Kusumi, and M. Fujihira, Appl. Phys. Lett. 66, 3245 (1995).

${ }^{11}$ A. Ianoul, P. Burgos, Z. Lu, R. Taylor, and L. Johnston, Langmuir 19, 9246 (2003).

${ }^{12}$ A. Ianoul, M. Street, D. Grant, J. Pezacki, R. Taylor, and L. Johnston, Biophys. J. 87, 3525 (2004).

${ }^{13}$ A. Ianoul, D. Grant, Y. Rouleau, M. Bani, L. Johnston, and J. Pezacki, Nat. Chem. Biol. 1, 196 (2005).

${ }^{14}$ D. Vobornik, D. Banks, Z. Lu, C. Fradin, R. Taylor, and L. Johnston, Appl. Phys. Lett. 93, 163904 (2008).

${ }^{15}$ H. Muramatsu, N. Chiba, N. Yamamoto, K. Homma, T. Ataka, M. Shigeno, H. Monobe, and M. Fujihira, Ultramicroscopy 71, 73 (1998).

${ }^{16}$ M. Platkov, A. Tsun, L. Nagli, and A. Katzir, Appl. Phys. Lett. 92, 104104 (2008).
${ }^{17}$ S. Kirstein, M. Mertesdorf, and M. Schonhoff, J. Appl. Phys. 84, 1782 (1998)

${ }^{18}$ H. Muramatsu, N. Chiba, K. Homma, K. Nakajima, T. Ataka, S. Ohta, A Kusumi, and M. Fujihira, Thin Solid Films 273, 335 (1996).

${ }^{19}$ P. Lambelet, M. Pfeffer, A. Sayah, and F. Marquis-Weible, Ultramicroscopy 71, 117 (1998).

${ }^{20}$ A. Fragola, L. Aigouy, P. Y. Mignotte, F. Formanek, and Y. de Wilde, Ultramicroscopy 101, 47 (2004).

${ }^{21}$ M. Yazdanpanah, M. Hosseini, S. Pabba, S. Berry, V. Dobrokhotov, A Safir, R. Keynton, and R. Cohn, Langmuir 24, 13753 (2008).

${ }^{22}$ P. G. de Gennes, F. Brochard-Wyart, and D. Quere, Capillary and Wetting Phenomena, 1st ed., (Springer, New York, 2002), Chap. 6, p. 143.

${ }^{23}$ F.-J. Elmer and M. Dreier, J. Appl. Phys. 81, 7709 (1997).

${ }^{24}$ P. Burgos, Z. Lu, A. Ianoul, C. Hnatovsky, M.-L. Viriot, L. J. Johnston, and R. S. Taylor, J. Microsc. 211, 37 (2003)

${ }^{25}$ D. Sarid, Scanning Force Microscopy, Oxford Series in Optical and Imaging Sciences, revised ed. (Oxford University Press, New York, 1994).

${ }^{26}$ Y. T. Yang, D. Heh, P. Wei, W. Fann, M. Gray, and J. Hsu, J. Appl. Phys. 81, 1623 (1997)

${ }^{27}$ M. K. Ghatkesar, T. Braun, V. Barwich, J.-P. Ramseyer, C. Gerber, M. Hegner, and H. Lang, Appl. Phys. Lett. 92, 043106 (2008).

${ }^{28}$ R. Patois, P. Vairac, and B. Cretin, Appl. Phys. Lett. 75, 295 (1999).

${ }^{29}$ W. H. J. Rensen, N. F. van Hulst, A. Ruiter, and P. West, Appl. Phys. Lett. 75, 1640 (1999).

${ }^{30}$ M. Koopman, B. de Bakker, M. Garcia-Parajo, and N. F. van Hulst, Appl. Phys. Lett. 83, 5083 (2003). 